\title{
ĐIỀU KHIỂN BÁM THÍCH NGHI BỀN VŨ̉NG HỆ PHI TUYẾN CÓ THÀNH PHẦN BẤT ĐỊNH HÀM KHÔNG BỊ CHẶN
}

\author{
Nguyễn Doãn Phước ${ }^{1, *}$, Lê Thị Thu Hà \\ ${ }^{\text {I} Đ a ̣ i ~ h o ̣ c ~ B a ́ c h ~ k h o a ~ H a ̀ ~ N o ̣ ̂ i, ~} 1$ Đại Cồ Việt, Q. Hai Bà Trung, Hà Nội \\ ${ }^{2} Đ a ̣ i$ học Kỹ thuật Công nghiệp Thái Nguyên, đường 3/2 Tích Lương, Thái Nguyên \\ *Email: phuoc.nguyendoan899@gmail.com
}

Đến Tòa soạn: 3/3/2014; Chấp nhận đăng: 4/10/2014

\section{TÓM TÁ̀T}

Với lớp bài toán điều khiển bám đối tượng phi tuyến có thành phần bất định hàm số bị chặn trong mô hình, người ta thường nghĩ ngay tới phương pháp điều khiển trượt. Đây là một trong các phương pháp điều khiển mang tính bền vững rất cao với nhiễu và sai lệch mô hình. Tuy nhiên một trong những hạn chế cơ bản của phương pháp điều khiển này là hiệu ứng chattering của nó. Đã có nhiều phương pháp bổ sung thêm để hạn chế hiệu ứng này được giới thiệu trong thời gian qua. Chúng đều tập trung vào việc nhận dạng để bù bớt đi thành phần bất định trong hệ. Bài báo này trình bày một phương pháp thích nghi làm giảm hiệu ứng chattering đó mà không cần nhận dạng và điều khiển bù. Hơn thế nữa phương pháp đề xuất của bài báo còn áp dụng được cho cả trường hợp hệ phi tuyến có hàm bất định không thỏa mãn điều kiện bị chặn.

Tù khóa: điều khiển thích nghi, điều khiển trượt, điều khiển bền vững, hệ bất định hàm.

\section{1. ĐẶT VẤN ĐỀ}

Thường gặp trong thực tế ở những hệ phi tuyến SISO (một đầu vào-một đầu ra) là hệ có mô hình mô tả quan hệ giữa tín hiệu vào $u(t)$ và tín hiệu ra $y(t)$ dưới dạng phương trình vi phân $[1,2,3]$ :

$$
y^{(n)}=f^{\prime}\left(u, y, \dot{y}, \ldots, y^{(n-1)}\right)+\Delta f+\eta(t)
$$

trong đó $\Delta f$ là sai lệch mô hình và $\eta(t)$ là nhiễu tác động vào hệ thống.

Điều đặc biệt là lớp hệ có dạng mô hình vào ra (1) ở trên luôn có thể chuyển được về cấu trúc mô hình trạng thái truyền ngược như sau $[4,5,2]$ :

$$
\left\{\begin{array}{l}
\dot{x}_{k}=x_{k+1} \quad \text { khi } 1 \leq k \leq n-1 \\
\dot{x}_{n}=f(\boldsymbol{x})+\varphi(\boldsymbol{x}, t)+u
\end{array}\right.
$$


với $\boldsymbol{x}=\left(x_{1}, \ldots, x_{n}\right)^{T}$ là vector trạng thái của hệ có tín hiệu đầu ra là $y=x_{1}, f(\boldsymbol{x})$ là hàm mô tả hệ thống được xác định từ hàm $f^{\prime}(\cdot)$ trong $(1)$ và $\varphi(x, t)$ là thành phần bất định của mô hình có liên quan tới sai lệch mô hình $\Delta f$ và nhiễu $\eta(t)$ mô tả trong (1).

Bài toán điều khiển bám ổn định cho lớp hệ (1) hoặc (2) được đặt ra ở đây là phải xác định được bộ điều khiển sao cho không phụ thuộc vào thành phần bất định không xác định được trong mô hình (2) là $\varphi(x, t)$ hoặc $\Delta f, \eta(t)$ trong (1), song hệ luôn có quỹ đạo trạng thái $\boldsymbol{x}(t)$ bám tiệm cận theo được quỹ đạo mẫu $\boldsymbol{x}_{d}(t)$ cho trước.

Tất nhiên ở bài toán điều khiển bám nêu trên cần phải có điều kiện kèm theo cho quỹ đạo đặt trước $\boldsymbol{x}_{d}(t)$, vì hệ không thể bám theo được mọi quỹ đạo bất kỳ. Một quỹ đạo $\boldsymbol{x}_{d}(t)$ được gọi là hợp lệ cho bài toán, nếu tồn tại ít nhất một tín hiệu đặt $\boldsymbol{u}_{d}(t)$ để nó là nghiệm của (2) ứng với tín hiệu đặt đó. Dễ thấy là các quỹ đạo trạng thái $\boldsymbol{x}_{d}(t)$ mong muốn phù hợp với dạng mô hình truyền ngược (2) đó phải là:

$$
\boldsymbol{x}_{d}=\left(w, \dot{w}, \ldots, w^{(n-1)}\right)
$$

trong đó $w(t)$ là hàm khả vi $n$ lần cho trước, $w^{(k)}$ là ký hiệu của đạo hàm bậc $k$ của $w(t)$.

Đã có khá nhiều phương pháp tìm nghiệm bài toán điều khiển trên được đề xuất. Phổ biến là những phương pháp có sử dụng nguyên tắc xấp xỉ hay nhận dạng thành phần bất định $\varphi(x, t)$ trong mô hình để bù nó bằng phản hồi trạng thái (0). Công cụ để xây dựng khối nhận dạng thành phần bất định đó thường là hệ mờ hoặc mạng neural [1].

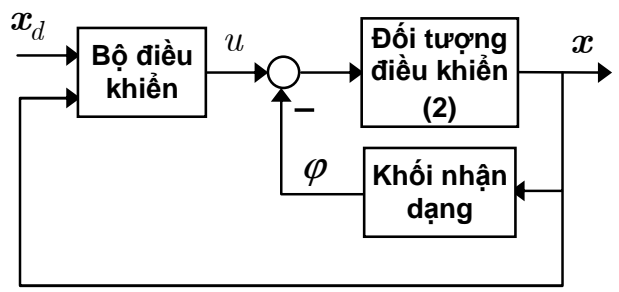

Hình 1. Cấu trúc điều khiển bù bất định.

Một phương pháp điều khiển khác không cần sử dụng khối nhận dạng là điều khiển trượt $[2,3]$. Đây là phương pháp điều khiển bền vững đảm bảo luôn có được điều kiện bám ổn định:

$$
\lim _{t \rightarrow \infty}|e(t)|=0 \text { và }|e(t)|<\infty, \forall t
$$

với $\boldsymbol{e}=\boldsymbol{x}_{d}-\boldsymbol{x}$ là sai lệch bám, mà không cần phải nhận dạng và bù thành phần bất định $\varphi(x, t)$. Tuy nhiên phương pháp này cần có giả thiết về tính bị chặn của thành phần bất định:

$$
\|\varphi(x, t)\|_{\infty}=\sup _{x, t}|\varphi(x, t)|=\delta_{\varphi}<\infty
$$

cũng như hiện tuợng rung (chattering) sinh ra khi bộ điều khiển phải giữ được sai lệch quỹ đạo trạng thái $e(t)$ ở lại trên mặt trượt, được hiểu là mặt: 


$$
\begin{aligned}
s(e) & =a_{1} e+a_{2} \dot{e}+\ldots+a_{n-1} e^{(n-2)}+e^{(n-1)} \\
& =\boldsymbol{a}^{T} \boldsymbol{e}
\end{aligned}
$$

có vector $\boldsymbol{a}=\left(a_{1}, \ldots, a_{n-1}, 1\right)^{T}$ với các hệ số là những số dương tùy chọn để đa thức:

$$
p(\alpha)=a_{1}+a_{2} \alpha+\cdots+a_{n-1} \alpha^{n-2}+\alpha^{n-1}
$$

là Hurwitz (có tất cả các nghiệm nằm bên trái trục ảo). Khi đã giữ lại được ở trên mặt trượt thì từ tính Hurwitz của đa thức (7), sai lệch $e(t)$ sẽ tự trượt trên đó về gốc $\mathbf{0}$.

Điều khiển trượt có ưu điểm là có tính bền vững cao với thành phần bất định hàm $\varphi(x, t)$, nhưng như đã nói, điều ta phải trả giá là nó lại sinh ra hiện tượng rung mà ở đó tín hiệu điều khiển $u(t)$ phải có tần số thay đổi giá trị rất cao để có thể giữ được quỹ đạo sai lệch $e(t)$ ở lại trên mặt trượt (6), thậm chí tần số này lại là vô cùng. Chuẩn (5) của thành phần bất định hàm càng lớn, biên độ dao động của $u(t)$ trạng thái chattering càng mạnh.

Để khắc phục nhược điểm trên của từng phương pháp điều khiển bù bất định và trượt, bài báo này sẽ trình bày một phương pháp điều khiển được xây dựng dựa trên nền điều khiển trượt để có tính bền vững với thành phần bất định $\varphi(x, t)$ và chỉnh định thích nghi cho tính không bị chặn của nó. Phương pháp này còn có thể làm giảm được cả hiện tượng rung mà không cần tới việc phải sử dụng thêm khâu nhận dạng và bù bất định. 0 biểu diễn nguyên tắc làm việc của phương pháp điều khiển của bài báo.

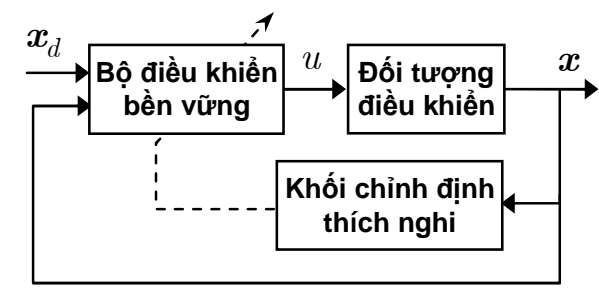

Hình 2. Cấu trúc điều khiển thích nghi bền vững.

\section{NỘI DUNG CHÍNH}

Trước tiên ta xét hệ bất định bậc $n$ có cấu trúc truyền ngược:

$$
\left\{\begin{array}{l}
\dot{x}_{k}=x_{k+1} \quad \text { khi } 1 \leq k \leq n-1 \\
\dot{x}_{n}=\varphi_{f}(\boldsymbol{x}, t)+\varphi_{g}(\boldsymbol{x}, t) u
\end{array}\right.
$$

với $\varphi_{f}(x, t)$ và $\varphi_{g}(x, t)$ là hai thành phần bất định không cần bị chặn của mô hình (hai hàm không biết trước). Tính không bị chặn của hai hàm $\varphi_{f}(x, t), \varphi_{g}(x, t)$ cũng là điểm khác biệt so với hệ (1).

Mặc dù là hai hàm bất định, song ta cũng cần một số giả thiết cho chúng. Giả thiết đầu tiên để hệ là điều khiển được là:

$$
\varphi_{g}(\boldsymbol{x}, t) \neq 0, \forall \boldsymbol{x}, t
$$


hoặc ít nhất nó cũng phải là tự dò được về gốc (zero detectable), tức là khi hệ đang ở điểm trạng thái không điều khiển được:

$$
\boldsymbol{x} \in \mathcal{Z}=\left\{\boldsymbol{x}\left(t_{0}\right) \in \mathbb{R}^{n} \mid \boldsymbol{\varphi}_{g}\left(\boldsymbol{x}, t_{0}\right)=0\right\}
$$

thì quỹ đạo trạng thái tự do của hệ vẫn bị chặn và có hướng tiến về gốc tọa độ. Nói cách khác, theo tinh thần nội dung định lý đảo Lyapunov thì phải tồn tại hàm $V(\boldsymbol{x}, t)$ hợp thức (bị chặn bởi hai hàm lớp $\mathcal{K}$ ) sao cho [5]:

$$
\frac{\partial V}{\partial t}+\frac{\partial V}{\partial \boldsymbol{x}}\left(\begin{array}{c}
x_{2} \\
\vdots \\
x_{n} \\
\varphi_{f}(\boldsymbol{x}, t)
\end{array}\right) \leq-\gamma(|x|) \text { khi } \boldsymbol{x} \in \mathcal{Z}
$$

trong đó $\gamma(\cdot)$ cũng là một hàm thuộc lớp $\mathcal{K}$.

Tiếp theo, ta giả thiết các thành phần bất định hàm không bị chặn $\varphi_{f}(x, t), \varphi_{g}(x, t)$ có cấu trúc:

$$
\begin{aligned}
& \varphi_{f}(\boldsymbol{x}, t)=\boldsymbol{\theta}_{f}^{T} \boldsymbol{f}(\boldsymbol{x}, t)+d(\boldsymbol{x}, t) \\
& \boldsymbol{\varphi}_{g}(\boldsymbol{x}, t)=\theta_{g}^{T} \boldsymbol{g}(\boldsymbol{x}, t)
\end{aligned}
$$

trong đó $\boldsymbol{f}(\boldsymbol{x}, t), \boldsymbol{g}(\boldsymbol{x}, t)$ là hai vector hàm rõ (đã biết), không cần có cùng số chiều với hệ và cũng không cần phải bị chặn, $\theta_{f}, \theta_{g}$ là hai vector tham số hằng số bất định và chỉ có riêng $d(\boldsymbol{x}, t)$ là thành phần tạp nhiễu (hàm) bất định thỏa mãn điều kiện bị chặn theo nghĩa có chuẩn vô cùng hữu hạn:

$$
\|d(\boldsymbol{x}, t)\|_{\infty}=\delta<\infty
$$

Có thể thấy giả thiết (9) này là luôn được thỏa mãn ở rất nhều hệ phi tuyến trong thực tế, chẳng hạn như các hệ robot, hệ cơ điện tử $[1,2]$.... Bài toán điều khiển bám cho hệ $(8)$ được hiểu là phải điều khiển sao cho quỹ đạo trạng thái $\boldsymbol{x}(t)$ của hệ bám tiệm cận theo được quỹ đạo mẫu $\boldsymbol{x}_{d}(t)$ cho bởi (3), tức là thỏa mãn điều kiện (4).

Định lý: Xét hệ truyền ngược bất định (8) tư dò được tới gốc. Dưới các giả thiết (9) và (10), hệ sẽ luôn điều khiển bám theo được tín hiệu mẫu (3) theo nghĩa (4) bằng bộ điều khiển động phản hồi trạng thái, có vector trạng thái:

$$
\left\{\begin{array}{l}
\dot{\hat{\boldsymbol{\theta}}}_{f}=-s(e) \mathbf{F}^{-1} \boldsymbol{f}(\boldsymbol{x}, t) \\
\dot{\hat{\boldsymbol{\theta}}}_{g}=-s(e) \mathbf{G}^{-1} \boldsymbol{g}(\boldsymbol{x}, t) u
\end{array}\right.
$$

và tín hiệu đầu ra:

$$
u=\frac{\sum_{k=1}^{n-1} a_{k} e^{(k)}+w^{(n)}-\hat{\boldsymbol{\theta}}_{f}^{T} \boldsymbol{f}(\boldsymbol{x}, t)+\lambda \operatorname{sgn}(s)}{\widehat{\boldsymbol{\theta}}_{g}^{T} \boldsymbol{g}(\boldsymbol{x}, t)}
$$


trong đó $\lambda>0$ là hằng số tùy chọn thỏa mãn $\lambda>\delta, \mathbf{F}, \mathbf{G}$ là hai ma trận đối xứng xác định duoong cũng tùy chon.

Chíng minh:

Trước tiên ta thấy ngay là hệ (8) cùng các giả thiết (9), (10) sẽ viết lại được thành:

$$
\left\{\begin{array}{l}
\dot{x}_{k}=x_{k+1} \quad \text { khi } 1 \leq k \leq n-1 \\
\dot{x}_{n}=\boldsymbol{\theta}_{f}^{T} \boldsymbol{f}(\boldsymbol{x}, t)+d(\boldsymbol{x}, t)+\boldsymbol{\theta}_{g}^{T} \boldsymbol{g}(\boldsymbol{x}, t) u
\end{array}\right.
$$

Với cấu trúc truyền ngược chặt (13) như trên, ta thấy $\boldsymbol{x}_{d}(t)$ cho bởi (3) cũng thỏa mãn (13) với một tín hiệu điều khiển $u_{d}(t)$ thích hợp, nên nó đủ điều kiện là quỹ đạo trạng thái mẫu. Nói cách khác sẽ đủ để có (4) nếu có:

$$
\lim _{t \rightarrow \infty}\left(w^{(k-1)}-x_{k}\right)=0, \forall k=1, \ldots, n
$$

và

$$
\left|w^{(k-1)}-x_{k}\right|<\infty, \forall k
$$

Bây giờ ta sử dụng nguyên lý điều khiển trượt với mặt trượt (6). Theo nguyên lý điều khiển này thì từ giả thiết về tính Hurwitz của đa thức (7), nếu đã có $s(e)=0$, cũng sẽ có (4). Do đó chỉ còn phải chứng minh là bộ điều khiển (11), (12) sẽ mang đến cho hệ thống điều mong muốn $s(e) \rightarrow 0$.

Trước tiên, ta giả sử đã biết hai vector tham số bất định $\theta_{f}, \theta_{g}$ trong mô hình (13). Vậy thì với hàm xác định dương:

$$
V(s)=\frac{1}{2} s^{2}
$$

ta sẽ được cùng với công thức (12) khi mà ở đó các vector hằng $\theta_{f}, \theta_{g}$ được thay chỗ cho $\hat{\boldsymbol{\theta}}_{f}, \hat{\boldsymbol{\theta}}_{g}$ :

$$
\begin{aligned}
\dot{V} & =s \dot{s} \\
& =s\left(\sum_{k=1}^{n-1} a_{k} e^{(k)}+w^{(n)}-\dot{x}_{n}\right) \\
\Leftrightarrow \quad \dot{V} & =s\left[\sum_{k=1}^{n-1} a_{k} e^{(k)}+w^{(n)}-\left(\boldsymbol{\theta}_{f}^{T} \boldsymbol{f}(\boldsymbol{x}, t)+d(\boldsymbol{x}, t)+\boldsymbol{\theta}_{g}^{T} \boldsymbol{g}(\boldsymbol{x}, t) u\right)\right] \\
& =s[-d(\boldsymbol{x}, t)-\lambda \operatorname{sgn}(s)] \\
& =-s d(\boldsymbol{x}, t)-\lambda|s| \\
& \leq|s||d(\boldsymbol{x}, t)|-\lambda|s| \leq|s|(\delta-\lambda)<0
\end{aligned}
$$


Điều này nói rằng trong trường hợp $\hat{\theta}_{f}, \hat{\theta}_{g}$ được thay bởi $\theta_{f}, \theta_{g}$, bộ điều khiển tĩnh (12) đã làm hệ kín thỏa mãn điều kiện $s(e) \rightarrow 0$ và $|s(\boldsymbol{e})|<\infty$, tức là thỏa mãn điều kiện (4) để bám theo tín hiệu mẫu (3).

Chuyển sang trường hợp hệ có $\theta_{f}, \theta_{g}$ là vector hằng bất định. Ký hiệu sai lệch:

$$
\boldsymbol{\delta}_{f}=\hat{\boldsymbol{\theta}}_{f}-\boldsymbol{\theta}_{f} \text { và } \boldsymbol{\delta}_{g}=\hat{\boldsymbol{\theta}}_{g}-\boldsymbol{\theta}_{g}
$$

Khi đó, vì $\theta_{f}, \theta_{g}$ là những vector hằng nên:

$$
\dot{\hat{\theta}}_{f}=\dot{\delta}_{f}, \quad \dot{\hat{\theta}}_{g}=\dot{\delta}_{g}
$$

Suy ra, cùng với bộ điều khiển (12) có chứa các tham số hàm cần chỉnh định $\widehat{\theta}_{f}, \widehat{\theta}_{g}$ thì:

$$
\begin{aligned}
\dot{s} & =\sum_{k=1}^{n-1} a_{k} e^{(k)}+w^{(n)}-\dot{x}_{n} \\
& =\sum_{k=1}^{n-1} a_{k} e^{(k)}+w^{(n)}-\left[\boldsymbol{\theta}_{f}^{T} \boldsymbol{f}(\boldsymbol{x}, t)+d(\boldsymbol{x}, t)+\boldsymbol{\theta}_{g}^{T} \boldsymbol{g}(\boldsymbol{x}, t) u\right] \\
& =\sum_{k=1}^{n-1} a_{k} e^{(k)}+w^{(n)}-\left[\boldsymbol{\theta}_{f}^{T} \boldsymbol{f}(\boldsymbol{x}, t)+d(\boldsymbol{x}, t)+\left(\boldsymbol{\theta}_{g}^{T}-\hat{\boldsymbol{\theta}}_{g}^{T}\right) \boldsymbol{g}(\boldsymbol{x}, t) u+\hat{\boldsymbol{\theta}}_{g}^{T} \boldsymbol{g}(\boldsymbol{x}, t) u\right] \\
& =\left(\hat{\boldsymbol{\theta}}_{f}-\boldsymbol{\theta}_{f}\right)^{T} \boldsymbol{f}(\boldsymbol{x}, t)+\left(\hat{\boldsymbol{\theta}}_{g}-\boldsymbol{\theta}_{g}\right)^{T} \boldsymbol{g}(\boldsymbol{x}, t) u-d(\boldsymbol{x}, t)-\lambda \operatorname{sgn}(s) \\
& =\boldsymbol{\delta}_{f}^{T} \boldsymbol{f}(\boldsymbol{x}, t)+\boldsymbol{\delta}_{g}^{T} \boldsymbol{g}(\boldsymbol{x}, t) u-d(\boldsymbol{x}, t)-\lambda \operatorname{sgn}(s)
\end{aligned}
$$

Sử dụng hàm xác định dương, trơn, đơn điệu tăng theo $|s(\boldsymbol{e})|$ và không bị chặn:

$$
\widehat{V}\left(s, \boldsymbol{\delta}_{f}, \boldsymbol{\delta}_{g}\right)=\frac{1}{2} s^{2}+\frac{1}{2} \boldsymbol{\delta}_{f}^{T} \mathbf{F} \boldsymbol{\delta}_{f}+\frac{1}{2} \boldsymbol{\delta}_{g}^{T} \mathbf{G} \boldsymbol{\delta}_{g}
$$

trong đó $\mathbf{F}, \mathbf{G}$ là hai ma trận đối xứng xác định dương tùy chọn, ta sẽ có:

$$
\dot{\bar{V}}=s \dot{s}+\boldsymbol{\delta}_{f}^{T} \mathbf{F} \dot{\boldsymbol{\delta}}_{f}+\boldsymbol{\delta}_{g}^{T} \mathbf{G} \dot{\boldsymbol{\delta}}_{g}
$$

Thay $\dot{s}$ từ công thức (14) và $u$ từ công thức (12) vào biểu thức (15) ở trên, ta đi đến:

$$
\begin{aligned}
\dot{\bar{V}} & =s\left[\boldsymbol{\delta}_{f}^{T} \boldsymbol{f}(\boldsymbol{x}, t)+\boldsymbol{\delta}_{g}^{T} \boldsymbol{g}(\boldsymbol{x}, t) u-d(\boldsymbol{x}, t)-\lambda \operatorname{sgn}(s)\right]+\boldsymbol{\delta}_{f}^{T} \mathbf{F} \dot{\boldsymbol{\delta}}_{f}+\boldsymbol{\delta}_{g}^{T} \mathbf{G} \dot{\boldsymbol{\delta}}_{g} \\
& =s\left[\boldsymbol{\delta}_{f}^{T} \boldsymbol{f}(\boldsymbol{x}, t)+\boldsymbol{\delta}_{g}^{T} \boldsymbol{g}(\boldsymbol{x}, t) u-d(\boldsymbol{x}, t)-\lambda \operatorname{sgn}(s)\right]+\boldsymbol{\delta}_{f}^{T} \mathbf{F} \dot{\overrightarrow{\boldsymbol{\theta}}}_{f}+\boldsymbol{\delta}_{g}^{T} \mathbf{G} \dot{\hat{\boldsymbol{\theta}}}_{g} \\
\Leftrightarrow \quad \dot{\bar{V}} & =-s d(\boldsymbol{x}, t)-s \boldsymbol{\lambda} \operatorname{sgn}(s)+\boldsymbol{\delta}_{f}^{T}\left(s \boldsymbol{f}(\boldsymbol{x}, t)+\mathbf{F} \dot{\hat{\boldsymbol{\theta}}}_{f}\right)+\boldsymbol{\delta}_{g}^{T}\left(s \boldsymbol{g}(\boldsymbol{x}, t) u+\mathbf{G} \dot{\overline{\boldsymbol{\theta}}}_{g}\right)
\end{aligned}
$$

Do đó, khi sử dụng cơ cấu chỉnh định (11) làm vector trạng thái cho bộ điều khiển xác định theo công thức (12) ta sẽ có tính xác định âm theo $s(e)$ của:

$$
\begin{aligned}
\dot{\bar{V}}\left(s, \boldsymbol{\delta}_{f}, \boldsymbol{\delta}_{g}\right) & =-s d(\boldsymbol{x}, t)-s \lambda \operatorname{sgn}(s) \\
& \leq|s|(\boldsymbol{\delta}-\lambda)<0
\end{aligned}
$$

và đó là điều kiện đủ để được $s(e) \rightarrow 0$. 
0 mô tả cấu trúc hệ thống điều khiển mô tả trong định lý. Ở đó các vector trạng thái $\widehat{\theta}_{f}, \widehat{\theta}_{g}$ của bộ điều khiển trượt (12) đã được biểu diễn tách ra khỏi bộ điều khiển thành khối chỉnh định thích nghi để làm rõ hơn nữa tính thích nghi của hệ.

Tuy nhiên cũng có điều cần bàn thêm ở đây là mặc dù đã có giả thiết hệ (8) là điều khiển được (hay ít nhất là tự dò được tới gốc), tức là phải có $\theta_{g}^{T} \boldsymbol{g}(\boldsymbol{x}, t) \neq 0$ với mọi $t$ và với mọi $\boldsymbol{x} \notin \mathcal{Z}$, song do ở bộ điều khiển ta đã thay vector $\boldsymbol{\theta}_{g}$ không biết trước bởi vector hàm $\hat{\theta}_{g}(t)$ được lấy từ cơ cấu chỉnh định thích nghi (11) nên vẫn có thể xảy ra trường hợp $\hat{\theta}_{g}\left(t_{k}\right)=\mathbf{0}$ ở một vài thời điểm $t_{k}$. Điều này sẽ làm cho tín hiệu ra $u(t)$ của bộ điều khiển (12) trở thành vô cùng tại đó. Để tránh trường hợp bất tiện này, ta có thể thay giá trị $\widehat{\theta}_{g}\left(t_{k}\right)=\mathbf{0}$ đó bằng hằng số rất nhỏ nhưng khác $\mathbf{0}$ của nó, chẳng hạn như:

$$
\hat{\boldsymbol{\theta}}_{g}(t)=\left\{\begin{array}{l}
\hat{\boldsymbol{\theta}}_{g}(t) \text { khi }\left|\hat{\boldsymbol{\theta}}_{g}(t)\right| \geq \boldsymbol{\varepsilon} \\
\boldsymbol{\varepsilon} \operatorname{sgn} \hat{\boldsymbol{\theta}}_{g}(t) \text { khi }\left|\hat{\boldsymbol{\theta}}_{g}(t)\right|<\boldsymbol{\varepsilon}
\end{array}\right.
$$

trong đó hàm lấy dấu của một vector được hiểu là:

$$
\operatorname{sgn}\left(\begin{array}{c}
v_{1}(t) \\
\vdots \\
v_{n}(t)
\end{array}\right)=\left(\begin{array}{c}
\operatorname{sgn} v_{1}(t) \\
\vdots \\
\operatorname{sgn} v_{n}(t)
\end{array}\right)
$$

\section{VÍ DỤ MINH HỌA}

Xét hệ truyền ngược (8) bậc 4 với các hàm bất định không bị chặn có cấu trúc cho bởi (9), trong đó:

$$
\boldsymbol{f}(\boldsymbol{x}, t)=\left(x_{2}, x_{3}, x_{4}\right)^{T}, \boldsymbol{g}(\boldsymbol{x}, t)=1 \text { và } \boldsymbol{\delta}=\|d\|_{\infty}=0.1
$$

Sử dụng bộ điều khiển (11), (12), (16) có các tham số:

$$
\begin{aligned}
& \mathbf{F}=0.2 \mathbf{I}_{3} \text { với } \mathbf{I}_{3} \text { là ma trận đơn vị của } \mathbb{R}^{3 \times 3} \\
& \mathbf{G}=100, \lambda=0.2, \theta_{f} \in \mathbb{R}^{3}, \theta_{g} \in \mathbb{R},
\end{aligned}
$$

và $\quad a_{1}=125, a_{2}=75, a_{3}=15, \varepsilon=0.005$

để điều khiển đối tượng (17), ta thu được kết quả mô phỏng cho ở 0 . Cụ thể $0 \mathrm{a}$ ) là đồ thị $w(t)=\sin (0.1 t)$ tạo thành trạng thái mẫu $\boldsymbol{x}_{d}(t)$ theo công thức (3) so sánh với tín hiệu ra $y=x_{1}(t)$ trong số các phần tử của quỹ đạo trạng thái thực $\boldsymbol{x}(t)$ của hệ, $0 \mathrm{~b}$ ) là các đồ thị của 3 phần tử trong vector trạng thái $\hat{\theta}_{f}(t)$ và $0 \mathrm{c}$ ) là đồ thị của trạng thái $\hat{\theta}_{g}(t)$ của bộ điều khiển thích nghi bền vững (11), (12). Từ đồ thị kết quả mô phỏng ta có thể thấy chỉ sau khoảng $60 \mathrm{~s}$ quỹ đạo trạng thái $\boldsymbol{x}(t)$ của hệ đã bám theo đúng được quỹ đạo mẫu $\boldsymbol{x}_{d}(t)$ và hệ đã đi vào chế độ xác lập với $\hat{\theta}_{f}(t), \hat{\theta}_{g}(t)$ khi đó là hằng số. 

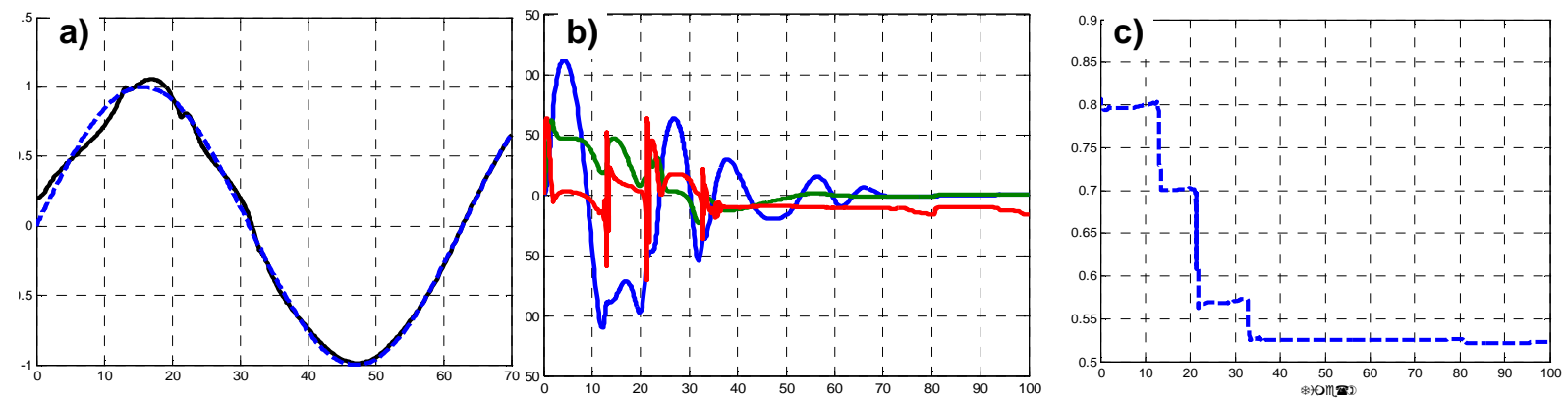

Hình 3. Kết quả mô phỏng hệ điều khiển thích nghi bền vững với quỹ đạo trạng thái mẫu so sánh với quỹ đạo trạng thái thực của hệ và các quỹ đạo trạng thái của bộ điều khiển

\title{
3. KẾT LUẬN
}

Tốc độ bám của hệ thích nghi bền vững tạo bởi bộ điều khiển (11), (12) là hoàn toàn chỉnh định được. Nếu chọn $\mathbf{F}, \mathbf{G}$ có $\|\mathbf{F}\|,\|\mathbf{G}\|$ càng nhỏ, tốc độ chỉnh định càng nhanh. Tương tự khi chọn $a_{1}, \ldots, a_{n-1}$ để đa thức Hurwitz (7) có nghiệm nằm càng xa trục ảo về phía trái, tốc độ bám của hệ cũng càng cao.

\section{TÀI LIỆU THAM KHẢO}

1. Hà L.T.T. (2012): Một số giải pháp nâng cao chất lượng hệ truyền động có khe hở trên cơ sở điều khiển thích nghi, bền vững. Luận án TS, Đại học Thái Nguyên.

2. Phước N.D. (2012): Phân tích và điều khiển hệ phi tuyến. NXB Bách khoa.

3. Utkin V. (1992): Sliding Modes in Optimization and Control. Springer Verlag New York.

4. Krstic M.; Kanellakopoulos,I.; Kokotovic,P. (1995): Nonlinear and Adaptive Control Design. John Wiley \& Sohn Inc.

5. Sastry S. (1999): Nonlinear System: Analysis, Stability and Control. Springer Verlag, New York, Inc.

\section{ABSTRACT}

ADAPTIVE AND ROBUST CONTROL OF NONLINEAR SYSTEMS WITH UNBOUNDED UNCERTAIN FUNCTIONS

\author{
Nguyen Doan Phuoc ${ }^{1, *}$, Le Thi Thu $\mathrm{Ha}^{2}$ \\ ${ }^{1}$ Hanoi University of Science and Technology, 1 Dai Co Viet, Hai Ba Trung, Hanoi \\ ${ }^{2}$ Thai Nguyen University of Technology, 3/2 Tich Luong Rd., ThaiNguyen City \\ *Email: phuoc.nguyendoan899@gmail.com
}


For the efficient solution of tracking control problems of a class of nonlinear systems with the bounded perturbation is sliding mode control the often used method. This method is known as the appropriate robust control technique to suppress the effect of bounded noises or disturbances in systems. However the occurred chattering of this control method is the main uncomfortable phenomenon in the close loop system. Many additional control techniques are published in few last years to damp this effect. They are primary based on approximation or identification of uncertainties to compensate their presence in the system. This paper proposes an approach to suppress this not wanted effect without using of the identification and compensation. Moreover the proposed control method can be also applied for tracking control of nonlinear systems with the presence of unbounded disturbances instead of bounded ones.

Keywords: adaptive control, sliding mode control, robust control, perturbed system. 